\title{
Crystal structure of histidine-containing phosphocarrier protein from Thermoanaerobacter tengcongensis MB4 and the implications for thermostability
}

\author{
FENG ChunYan ${ }^{1,2}$, GAO Feng ${ }^{3}$, LIU YiWei ${ }^{4}$, WANG GuiHua ${ }^{1}$, PENG Hao ${ }^{1}$, MA YanHe $^{5}$, \\ YAN JingHua ${ }^{1} \&$ GAO George $\mathrm{Fu}^{1,2,6^{*}}$ \\ ${ }^{1}$ CAS Key Laboratory of Pathogenic Microbiology and Immunology, Institute of Microbiology, Chinese Academy of Sciences, \\ Beijing 100101, China; \\ ${ }^{2}$ Graduate University of Chinese Academy of Sciences, Beijing 100049, China; \\ ${ }^{3}$ Institute of Biophysics, Chinese Academy of Sciences, Beijing 100101, China; \\ ${ }^{4}$ Laboratory of Structural Microbiology, Institute of Microbiology, Chinese Academy of Sciences, Beijing 100101, China; \\ ${ }^{5}$ State Key Laboratory of Microbial Resources, Institute of Microbiology, Chinese Academy of Sciences, Beijing 100101, China; \\ ${ }^{6}$ Beijing Institutes of Life Science, Chinese Academy of Sciences, Beijing 100101, China
}

Received April 11, 2011; accepted April 17, 2011

\begin{abstract}
Protein thermostability is an inherent characteristic of proteins from thermophilic microorganisms, and therefore enables these organisms to survive at extreme temperatures. Although it is well-known that thermostable proteins are critical for the growth of thermophilic organisms, the structural basis of protein thermostability is not yet fully understood. The histidine-containing phosphocarrier (HPr) protein, a phosphate shuttle protein in the phosphoenolpyruvate-dependent sugar transport system (PTS) of bacterial species, is an ideal model for investigating protein thermostability with respect to its small size and deficiency in disulphide bonds or cofactors. In this study, the HPr protein from Thermoanaerobacter tengcongensis (TtHPr) is cloned and purified. Crystal structure with good quality has been determined at $2.3 \AA$ resolution, which provides a firm foundation for exploring the thermostable mechanism. However, it shows that the crystal structure is conserved and no clue can be obtained from this single structure. Furthermore, detailed comparison of sequence and structure with the homologs from meso- or thermophilic bacteria shows no obvious rule for thermostability, but the extra salt-bridge existing only in thermophilic bacteria might be a better explanation for thermostability of HPr. Thus, mutations are performed to interrupt the salt-bridge in HPrs in thermophilic bacteria. Using site-directed mutations and the circular dichroism method, thermostability is evaluated, and the mutational variations are shown to have a faster denaturing rate than for wild-type viruses, indicating that mutations cause instability in the HPrs. Understanding the higher-temperature resistance of thermophilic and hyperthermophilic proteins is essential to studies on protein folding and stability, and is critical in engineering efficient enzymes that can work at a high temperature.
\end{abstract}

HPr, crystal structure, thermostability, salt-bridge, Thermoanaerobacter tengcongensis

Citation: Feng C Y, Gao F, Liu Y W, et al. Crystal structure of histidine-containing phosphocarrier protein from Thermoanaerobacter tengcongensis MB4 and the implications for thermostability. Sci China Life Sci, 2011, 54: 513-519, doi: 10.1007/s11427-011-4182-x

Thermoanaerobacter tengcongensis, with an optimal growth temperature of $75^{\circ} \mathrm{C}$, belongs to the thermophile

*Corresponding author (email: gaof@im.ac.cn) class of extremophilic organisms [1,2]. As an inherent property, proteins derived from these thermophilic species display exceptionally high thermostability, but how these proteins deal with high temperatures and produce optimal 
activity under such extreme conditions is currently unknown. A large number of studies have been performed to unravel the molecular mechanisms of protein thermostability. However, to date, no universal paradigm has been presented and thermostable proteins are known to resist high temperatures in diverse ways [3]. Several hypotheses have been proposed, including relatively small solvent-exposed surface area [4], improved atom packing and electrostatic interactions [5-7], increased hydrogen bond networks [8-11] and internal polar contacts in a monomeric structure [12], proper protein flexibility [13], surface salt bridges and quaternary structures [14], and amino acids composition [15]. Indeed, theoretical and experimental research of protein thermostability mechanisms is still under investigation. Proper understanding of the molecular basis of thermal adaptation will, no doubt, be essential for studies relating to protein folding and protein interaction mechanisms, leads to the development of more efficient protein engineering strategies, and as a consequence, improves biotechnological processes.

The histidine-containing phosphocarrier protein (HPr) from Thermoanaerobacter tengcongensis is a small-size ( 88 amino acids) monomeric protein, which acts as a phosphate shuttle in the phosphoenolpyruvate-dependent sugar transport system (PTS) of bacterial species [16]. The protein $\mathrm{HPr}$ can be phosphorylated at $\mathrm{N}^{\delta 1}$ of His 15 by PTS enzyme I (EI) and transfer this phosphate group to $\mathrm{N}^{\varepsilon 2}$ of a histidine residue of the EIIA domain of the enzyme II (EII) complex [17,18], and therefore guarantee the uptake and phosphorylation of many absorbed sugars including glucose, fructose, mannitol and mannose, for several bacterial metabolic, sensory, and regulatory pathways [19]. Without the presence of extra affecting factors such as disulfides or prosthetic groups, HPrs from diverse thermo-resistant habitats appear to be good model systems for the elucidation of mechanisms responsible for protein thermal adaptation [20].

Structures of HPr proteins from a variety of organisms including Escherichia coli, Enterococcus faecalis, Bacillus subtilis, Streptococcus faecalis, Bacillus stearothermophilus, Staphylococcus auras, and Mycoplasma capricolum, have been determined by X-ray crystallography [9,21-24] or NMR spectrometry [25,26]. Despite large variations in the primary structure of different HPrs, the secondary structural elements together with the ternary structures are wellconserved. The classic structure of HPr consists of a fourstranded antiparallel $\beta$-sheet and three $\alpha$-helices arranged in a $\beta \alpha \beta \beta \alpha \beta \alpha$ open $\beta$-sandwich topology. The numerous three-dimensional structures presently available in the Protein Data Bank (PDB), providing a large amount of high quality statistical information, enable us to perform a detailed sequence and structure comparison of these HPrs and determine the cause of the existing thermostability differences.

In this study, Thermoanaerobacter tengcongensis $\mathrm{HPr}$ (TtHPr) was cloned and over-expressed. High purity of
Tt HPr was obtained by heat inactivation at $70^{\circ} \mathrm{C}$ based on $T t \mathrm{HPr}$-specific thermostability [27] and the crystal structure of the most thermostable $T t \mathrm{HPr}$ was determined at $2.3 \AA$ resolution. Based on the structural results, a detailed comparison of structural features between the proteins of different habitat temperatures was performed. Interestingly, we found that HPrs from the thermo-organisms have an extra salt-bridge other than HPrs from meso-organisms, which might contribute to the thermostability of this protein. The variations with an interrupted salt-bridge were checked for thermostability by circular dichroism (CD) spectroscopy; the results proved that the salt-bridge was a good explanation for thermostability. The present findings are anticipated to help in the design of highly efficient enzymes that can work at high temperatures in modern biotechnology and to contribute to the understanding of the mechanisms underlying microbial evolution.

\section{Materials and methods}

\subsection{Protein clone, mutation, expression and purifica- tion}

The genes of $T t \mathrm{HPr}$ and $B s t \mathrm{HPr}$ were cloned into the pET-30a vector from genomic DNA using standard molecular biology procedures. The K62T mutant ( $\mathrm{K}$ to $\mathrm{T}$ mutation at amino acid position 62) of $T t \mathrm{HPr}$ and $\mathrm{K} 57 \mathrm{~T}$ ( $\mathrm{K}$ to $\mathrm{T}$ mutation at amino acid position 57) of Bst HPr were constructed using the QuickChange mutagenesis kit and standard molecular biology techniques. All proteins were over-expressed in E. coli BL21 (DE3) cells which were grown at $37^{\circ} \mathrm{C}$ to an $A_{600}$ of $0.8-1.0$, and then induced with $1 \mathrm{mmol} \mathrm{L}^{-1}$ isopropyl- $\beta$-D-thiogalactopyranoside (IPTG). Cells were harvested by centrifugation and disrupted with sonication on ice. For wild-type HPrs, the mixture was

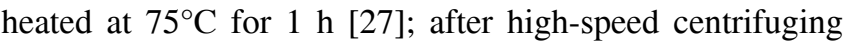
the resulting supernatant was applied to an anion-exchange resource Q (GE Healthcare), then a desalting column followed by purification on a Superdex 75 (Amersham Bioscience). The variants of $\mathrm{HPr}$ were purified at $4{ }^{\circ} \mathrm{C}$, first by nickel-affinity chromatography and then applied to the columns mentioned above.

\subsection{Crystallization, X-ray data collection, structure determination and refinement}

Crystallization experiments were performed using the hanging drop vapor diffusion method at $291 \mathrm{~K}$. After optimization, crystals with good diffraction were obtained at $277 \mathrm{~K}$ by mixing $2 \mu \mathrm{L}$ of protein solution $\left(10 \mathrm{mg} \mathrm{mL}^{-1}\right)$ and $2 \mu \mathrm{L}$ of reservoir solution (15\% PEG 20000, $0.1 \mathrm{~mol} \mathrm{~L}^{-1}$ MES, pH 6.5). X-ray diffraction data were collected on $R$-axis image plates. Data were processed and scaled with HKL 2000 [28]. 
The $T t \mathrm{HPr}$ structure was solved by molecular replacement using the program MOLREP [29] with the Bst HPr (PDB code 1Y4Y) as an initial search model. The model was subsequently refined in Refmac5 [30] and several iterative cycles of manual rebuilding were performed in COOT [31]. Consequently, PHENIX AutoBuild software [32] was used to deal with the twin crystal and fit the amino-acid sequence to the electron-density map. The protein structure quality was validated by PROCHECK [33]. Statistics of refinement and data collection are shown in Table 1. Atomic coordinates of HPr from Thermoanaerobacter tengcongensis have been deposited in the PDB (http://www.rcsb.org/pdb) under accession code $3 \mathrm{LNW}$.

\subsection{Structure analysis and comparison}

All HPrs from the PDB with similar native state were selected for structural comparison (1POH from E. coli and $E c \mathrm{HPr}, 1 \mathrm{PTF}$ from E. faecalis and EfHPr, $1 \mathrm{PCH}$ from $M$. capricolum, McHPr, and 1Y4Y from B. stearothermophilus, $E f H P r$, all to be mentioned below). Ion pair interactions were identified in the structure of HPrs according to the criterion suggested by Barlow and Thornton [34], of a distance less than or equal to $4 \AA$ between charged groups using the program CONTACT in the CCP4 suite [35]. The total

Table 1 Data collection and refinement statistics ${ }^{\text {a) }}$

\begin{tabular}{|c|c|}
\hline \multicolumn{2}{|l|}{ Data collection } \\
\hline Lattice type & Trigonal \\
\hline Space group & P31 \\
\hline Unit cell $\left(\AA 0^{\circ}\right)$ & $a=b=67.972, c=50.447 ; \alpha=\beta=90, \gamma=120$ \\
\hline Resolution $(\AA)^{*}$ & $38.30-2.30(2.38-2.30)$ \\
\hline No. of reflections & 63655 \\
\hline No. of unique reflections & 11574 \\
\hline Completeness $(\%)$ & $99.9(100)$ \\
\hline Multiplicity & $5.5(5.5)$ \\
\hline $\mathrm{I} / \sigma(\mathrm{I})$ & $24.3(8.4)$ \\
\hline$R_{\text {merge }}(\%)$ & $7.2(24.5)$ \\
\hline \multicolumn{2}{|l|}{ Refinement } \\
\hline$R_{\text {work }}(\%)$ & 19.3 \\
\hline$R_{\text {free }}(\%)$ & 22.3 \\
\hline \multicolumn{2}{|l|}{ Ramachandran plot } \\
\hline Residues in preferred regions & $95.7 \%$ \\
\hline Residues in allowed regions & $4.3 \%$ \\
\hline $\begin{array}{l}\text { Residues in disallowed } \\
\text { regions }\end{array}$ & $0 \%$ \\
\hline \multicolumn{2}{|l|}{ Average B-factor ( $\mathrm{\AA} 2)$} \\
\hline Main chains & 28.83 \\
\hline Side chains and waters & 33.30 \\
\hline Average B value for all atoms & 31.10 \\
\hline RMS B main-chain & 2.74 \\
\hline RMS B side-chain & 4.74 \\
\hline Total number of atoms & 2097 \\
\hline No. of water molecules & 135 \\
\hline \multicolumn{2}{|l|}{ RMS deviations } \\
\hline Bond lengths $(\AA)$ & 0.003 \\
\hline Bond angle $\left(^{\circ}\right)$ & 0.678 \\
\hline
\end{tabular}

a) $*$, Numbers in parentheses refer to data of the highest resolution shell. accessible surface areas (ASA) were also calculated with the program CCP4 suite with a probe radius of $1.4 \AA$. The program WHATIF [36] (http://swift.cmbi.ru.nl/servers/html/ index.html) was used to identify all hydrogen bonds in the structures. All figures representing protein structures were prepared by PyMol [37].

\subsection{Thermostability measurements with circular di- chroism}

The CD spectroscopy experiments of $T t \mathrm{HPr}$ were conducted using a Jasco J-810 automatic spectropolarimeter (Japan Spectroscopic, Tokyo, Japan), which permitted accurate temperature control. The optical path length was $10 \mathrm{~nm}$. The CD spectra of native protein were obtained using a concentration of $5 \mu \mathrm{mol} \mathrm{L} \mathrm{L}^{-1}$ with buffer $20 \mathrm{mmol} \mathrm{L}^{-1}$ Tris- $\mathrm{HCl}, \mathrm{pH} 8.0$ at $25^{\circ} \mathrm{C}$. Buffer scans were accumulated and subtracted from the sample scans, and the mean residue ellipticity was calculated.

The CD measurements of $T t \mathrm{HPr}$ thermostability and thermodynamics were obtained through analysis of the chemical (urea) and thermal denaturation curves, where wavelength $222 \mathrm{~nm}$ was used to examine the conformation of the protein, according to methods described previously [38]. Briefly, the $\mathrm{CD}$ temperature scans were performed by varying the temperature from 20 to $100^{\circ} \mathrm{C}$ at $0.5^{\circ} \mathrm{C}$ intervals. Protein solution equilibrated previously with $11 \mathrm{~mol} \mathrm{~L}^{-1}$ urea $23 \mathrm{~h}$ at room temperature were used as control of $100 \%$ denaturation. The fraction of denaturizing protein was calculated from the plotting changes in circular dichroism, based on the assumption that the unfolding equilibrium of protein follows a two-state mechanism. The resulting data points were curve-fitted using SigmaPlot (Jandel Scientific, CA, USA).

\section{Results and discussion}

\subsection{The structural quality of the refined $T t \mathrm{HPr}$ model}

The structure of $T t \mathrm{HPr}$ protein had been refined to an $R$-factor of 0.19 with good stereochemical geometries, as indicated in Table 1. TtHPr had crystallized under space group $P 3_{1}$ and contained three protein molecules in the asymmetric unit. The electron density map fitted well with the main chains and side chains of the final refined model. Judged by the Ramachandran plot [39] statistics and Rotamer analysis, the $T t \mathrm{HPr}$ structure was of good quality with $95.7 \%$ of the residues in the most favored regions, $4.26 \%$ of the residues in allowed regions and no residues in disallowed regions. Furthermore, the quality of the $2 F_{\mathrm{o}}-F_{\mathrm{c}}$ map was well-defined even under the level of $2.5 \sigma$. The structural data we had obtained from the crystal offered a good platform to reveal the reason why $T t \mathrm{HPr}$ was so thermostable. 


\subsection{Overall structure of $T t \mathrm{HPr}$}

The three-dimensional crystal structure of $T t \mathrm{HPr}$ was determined at a resolution of $2.3 \AA$, with crystallographic $R_{\text {work }}$ and $R_{\text {free }}$ factors of $19.3 \%$ and $22.3 \%$, respectively (Table 1 ). The overall fold of $T t \mathrm{HPr}$ was displayed as an open-faced $\beta$-sandwich with a $\beta_{1} \alpha_{1} \beta_{2} \beta_{3} \alpha_{2} \beta_{4} \alpha_{3}$ secondary structural arrangement of three $\alpha$-helices, $\alpha_{1(17-28)}, \alpha_{2(47-53)}, \alpha_{3(70-83)}$, overlaying a four-stranded $\beta$-sheet, $\beta_{1(2-7)}, \beta_{2(32-37)}, \beta_{3(40-43)}$, $\beta_{4(58-66)}$, which was very conserved in all the HPrs (Figure 1A). Although the asymmetric unit of the $T t \mathrm{HPr}$ crystal comprised three protein molecules, it showed the same monomeric form in solution as other HPrs and variants measured by FPLC (data not shown). The topology structure was also presented according to the crystal structure for easier understanding of the secondary structure of $T t \mathrm{HPr}$
(Figure 1B). From these results, no conclusion about the thermostability could be deduced by this single crystal structure, which indicated other methods, such as comparison of structures of homology available in the PDB, would need to be considered in further explorations.

\subsection{Structural comparison with other HPrs}

Thus, further structural comparison among the HPrs with similar native state available in PDB was conducted. Five HPrs $(E c \mathrm{HPr}, E f \mathrm{HPr}, M c \mathrm{HPr}$ and $B s t \mathrm{HPr})$ including $T t \mathrm{HPr}$ were selected, three of which were from mesophilic organisms $(E c \mathrm{HPr}, E f \mathrm{HPr}, M c \mathrm{HPr})$ and two of which (Bst $\mathrm{HPr}$ and $T t \mathrm{HPr}$ ) were from thermophilic organisms. The overall fold of $T t \mathrm{HPr}$ suggested a high similarity to that of its homologs from other organisms (Figure 2A). It should be noted that
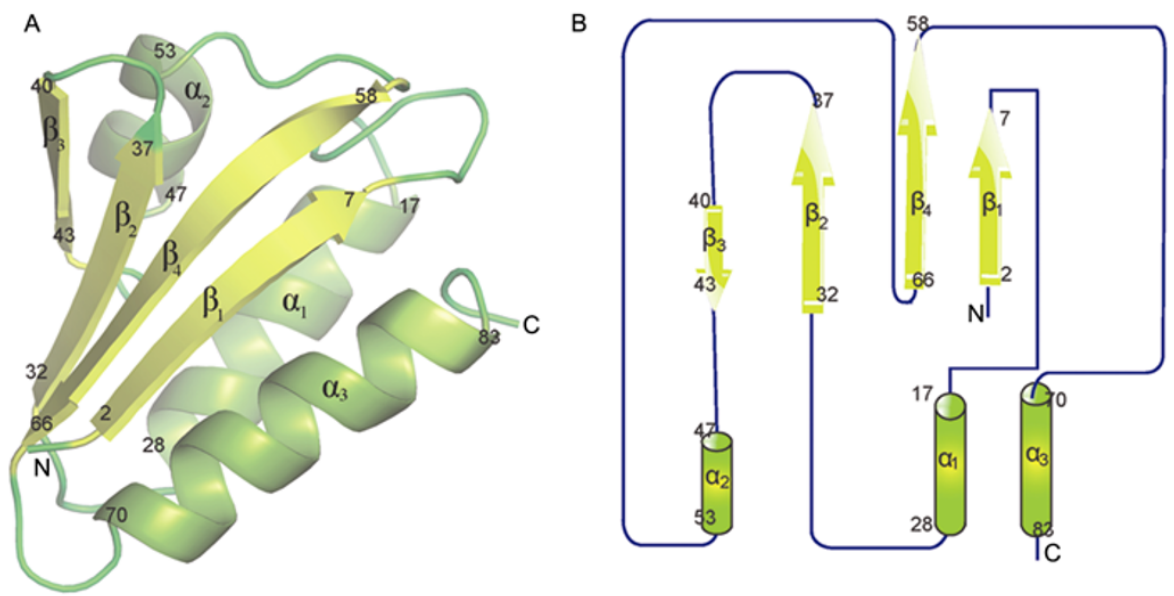

Figure 1 The overall structure of $T t \mathrm{HPr}$. A, Schematic diagram of $T t \mathrm{HPr}$. The secondary structural elements are marked by the apparent order in amino acid sequences and shown in different colors. B, The topology of TtHPr shows the arrangement of the secondary structure in TtHPr.

A

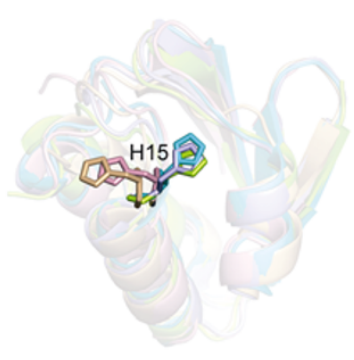

B

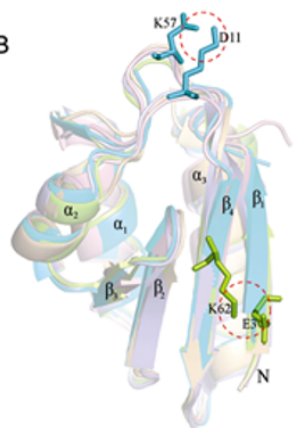

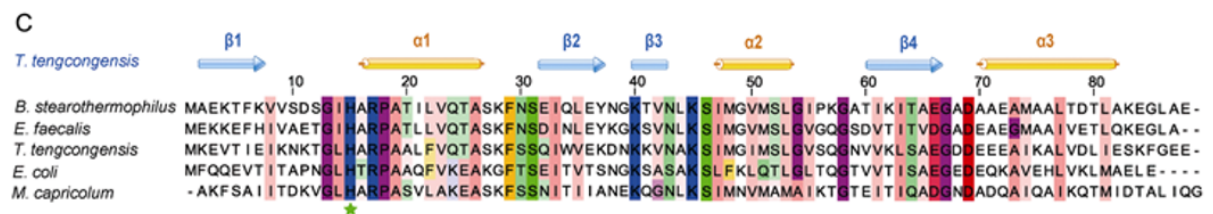

Figure 2 Comparison of the HPrs from meso- and thermo-organisms. A, Crystal structure comparison of the HPrs from meso- and thermo-organisms. Conserved $\mathrm{H} 15$ is shown in detail. The imidazole ring of residue His 15 is presented in two distinct conformations in five HPrs. The HPrs are color-coded as $1 \mathrm{POH}$, orange; 1PTF, green-cyan; $1 \mathrm{PCH}$, blue; $1 \mathrm{Y} 4 \mathrm{Y}$, light pink; 3LNW, lemon. B, The indication of the salt-bridges in HPrs. One red dashed circle indicates the salt-bridge between E3 and K62 in TtHPr, and the other red dashed circle indicates the salt-bridge D11 and K57 in BstHPr. C, Sequence alignments among $T t \mathrm{HPr}, B s t \mathrm{HPr}, E c \mathrm{HPr}, M c \mathrm{HPr}$, and $E f \mathrm{HPr}$. His 15 is indicated in green star. Conserved amino acids are marked in colors. 
the conformation of residues 11-18 among all aligned HPr structures displayed slight differences, whereas the highly conserved His15 and Ser46 are expected to be the phosphorylation site, as the case in other HPrs. The active site residue His 15 of HPr could assume two distinct conformations which were termed respectively OPEN and CLOSED, corresponding to two different protonation forms of the histidine ring [40].

To unravel the molecular mechanisms of protein thermostability, several paradigms published as potential mechanisms have been compared (Table 2). These paradigms included relatively small solvent-exposed surface area [4], improved atom packing and electrostatic interactions [5], increased hydrogen bond networks [8] and internal polar contacts in a monomeric structure [12], proper protein flexibility [13], surface salt bridges and quaternary structure [14], and amino acids composition [15]. Strikingly, we found that the HPr from thermostable organisms (Bst and $T t$ ) have an extra salt-bridge (Table 2 and Figure 2B), which did not show up in HPrs from meso-organism (E. coli, E. fae, M. cap). In $T t \mathrm{HPr}$, the side chain carboxyl atom of Asp was with $2.63 \AA$ from the nitrogen atom of Lys, which suggested a strong salt-bridge formed between E3 and K62. This salt-bridge might tighten the structure between fragment $\beta_{1}$ and $\beta_{4}$ contributing to the thermostability of TtHPr. Simultaneously, the side chain carboxyl atom of Glu was with $2.55 \AA$ from the nitrogen atom of Lys, which suggested a strong salt-bridge formed between D11 and K57 in Bst HPr. These extra salt-bridges might be a better explanation for thermostability of HPr from thermo-organisms.

After alignment of the amino acid sequence, we found that $T t \mathrm{HPr}$ showed gradually increasing sequence identities of $37.7 \%, 41.9 \%, 47.8 \%$, and $51 \%$ with $B s t \mathrm{HPr}, E c \mathrm{HPr}$, $\mathrm{McHPr}$, and EfHPr (Figure 2C). The amino acids forming salt-bridges were paid special attention. It was showed that in position 57, only Bst $\mathrm{HPr}$ has the $\mathrm{K}$, whereas in $T t \mathrm{HPr}$,
$E c \mathrm{HPr}$ and $E f \mathrm{HPr}$, it was appeared to be Q, and the $M c \mathrm{HPr}$ has a $\mathrm{T}$ in this position. Thus, to avoid the disturbance of the other factors, a K57 to T57 mutation was undertaken to interrupt the salt-bridge in $B s t \mathrm{HPr}$. In the same manner, the sequence alignment indicated that Bst $\mathrm{HPr}$ and $T t \mathrm{HPr}$ with thermostability possess the $\mathrm{K}$ in position 62 , whereas the $E c \mathrm{HPr}, M c \mathrm{HPr}$ and EfHPr from mesophilic organisms possess the T. With these considerations, the site directed mutation-K62T was mutated in TtHPr. Thus, two variations of HPrs with an interrupted salt-bridge were constructed to confirm the influence of the salt-bridge.

\subsection{Confirmation of the influence of the salt-bridge in the thermostability of HPrs by CD spectra}

The secondary structural features of $T t \mathrm{HPr}$ were examined by far-UV CD as depicted in Figure 3A. The CD spectra analysis suggested that native $T t \mathrm{HPr}$ contains typical $\alpha$-helices and $\beta$-strands, consistent with the final refined $T t \mathrm{HPr}$ structure. In the case of chemical denaturation, at least $90 \%$ reversibility was observed, and the transitions were independent of protein concentration over the studied concentration range of $5-50 \mathrm{mmol} \mathrm{L}^{-1}$. The present result showed that $50 \%$ of $T t \mathrm{HPr}$ remained stable at $8.4 \mathrm{~mol} \mathrm{~L}^{-1}$ urea, in agreement with the conclusion that thermostable enzymes were more resistant to physical and chemical denaturation [41]. This indicated that TtHPr might be the most thermostable HPr ever reported at present $[9,38]$.

To verify the influence of the salt-bridge in the thermostability of HPrs, site directed mutations were performed as described in a previous report [42]. Bst HPr (K57T variant) and $T t \mathrm{HPr}$ (K62T variant) with interrupted salt-bridges were constructed. Furthermore, the variants were purified and then the thermostability was measured using circular dichroism analysis. As shown in Figure 3C(a), the initial temperature of denaturation in wild-type Bst $\mathrm{HPr}$ was the

Table 2 Properties of HPr proteins from meso- or thermo-philic bacteria

\begin{tabular}{|c|c|c|c|c|c|}
\hline Property & $E c \mathrm{HPr}$ & EfHPr & $\mathrm{McHPr}$ & Bst $\mathrm{HPr}$ & $T t \mathrm{HPr}$ \\
\hline Optimal habitat temperature $\left({ }^{\circ} \mathrm{C}\right)$ & 37 & $10-42$ & $30-37$ & $55-65$ & 75 \\
\hline PDB code & $1 \mathrm{POH}$ & $1 \mathrm{PTF}$ & $1 \mathrm{PCH}$ & $1 Y 4 Y$ & 3LNW \\
\hline No. of residues in crystal structure & 85 & 87 & 88 & 87 & 86 \\
\hline Resolution & $2.0 \AA$ & $1.6 \AA$ & $1.8 \AA$ & $2.0 \AA$ & $2.3 \AA$ \\
\hline Sequence identity (with $T t \mathrm{HPr}$ ) & $41.86 \%$ & $51 \%$ & $37.7 \%$ & $47.73 \%$ & $100 \%$ \\
\hline No. of residues of three $\alpha$-helix & $35(41.2 \%)$ & $35(40.2 \%)$ & $32(36.3 \%)$ & $38(43.2 \%)$ & $33(38.4 \%)$ \\
\hline No. of residues of four $\beta$-sheet & $26(30.6 \%)$ & $26(29.9 \%)$ & $22(25 \%)$ & $22(25 \%)$ & $26(30.2 \%)$ \\
\hline Hydrogen bonds & 84 & 87 & 86 & 87 & 86 \\
\hline Salt bridge & 0 & 0 & 0 & 1 & 1 \\
\hline Charged residues (D, E, H, K, R) & $19(22.4 \%)$ & $22(25.3 \%)$ & $18(20.5 \%)$ & $21(23.9 \%)$ & $25(29.1 \%)$ \\
\hline Polar residues $(\mathrm{N}, \mathrm{Q}, \mathrm{S}, \mathrm{T})$ & $24(28.2 \%)$ & $18(20.7 \%)$ & $24(27.3 \%)$ & $19(21.6 \%)$ & $18(20.9 \%)$ \\
\hline Aromatic residues $(\mathrm{F}, \mathrm{Y}, \mathrm{W})$ & $4(4.7 \%)$ & $3(3.5 \%)$ & $2(2.3 \%)$ & $3(3.4 \%)$ & $4(4.7 \%)$ \\
\hline Apolar residues $(\mathrm{G}, \mathrm{A}, \mathrm{V}, \mathrm{L}, \mathrm{I})$ & $33(38.8 \%)$ & $39(44.8 \%)$ & $39(44.3 \%)$ & $40(45.5 \%)$ & $35(40.7 \%)$ \\
\hline B-branched residues $(\mathrm{I}, \mathrm{V}, \mathrm{T})$ & $20(23.6 \%)$ & $20(23.0 \%)$ & $23(26.1 \%)$ & $20(22.7 \%)$ & $18(20.9 \%)$ \\
\hline
\end{tabular}



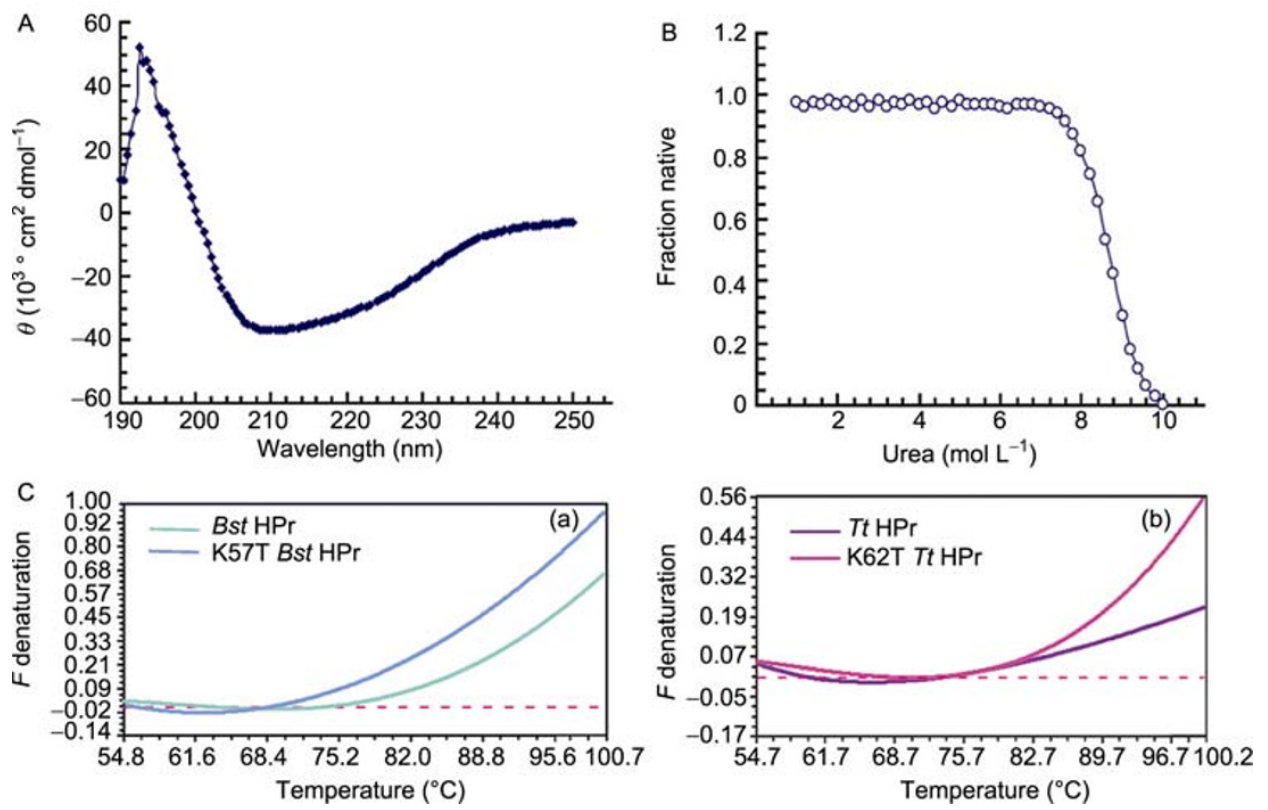

Figure 3 The secondary structure and thermostability evaluation analysis of HPrs by CD spectra. A, CD spectra of native $T t \mathrm{HPr}$, showing the typical characteristic of $\alpha+\beta$ protein. B, Urea denaturation curves for the TtHPr. The X-ray indicates the concentration of the urea and the Y-ray indicates the fraction of the native. C, Stability measurements for the HPrs and its variants with circular dichroism are as described in the text. The X-ray indicates the temperature and the Y-ray indicates the fraction of denaturation. The K62T variant of $T t \mathrm{HPr}$ shows the same decreased thermostability to wild-type $T t \mathrm{HPr}$ as K57T variant of $B s t \mathrm{HPr}$ to wild-type $B s t \mathrm{HPr}$.

same as the $\mathrm{K} 57 \mathrm{~T}$ variant of $B s t \mathrm{HPr}$ at about $68.4^{\circ} \mathrm{C}$. However, the K57T variant in Bst HPr showed much faster denaturing rate. At $100^{\circ} \mathrm{C}$, about $98 \% \mathrm{~K} 57 \mathrm{~T}$ variant was denatured compared with $68 \%$ for wild-type Bst $\mathrm{HPr}$, indicating that the $\mathrm{K} 57 \mathrm{~T}$ variant was less stable than wild-type $B s t \mathrm{HPr}$. In Figure $3 \mathrm{C}(\mathrm{b})$, the initial temperature of denaturation was about $76^{\circ} \mathrm{C}$ both in wild-type $T t \mathrm{HPr}$ and in $\mathrm{K} 62 \mathrm{~T}$ variant $T t \mathrm{HPr}$. At $100^{\circ} \mathrm{C}$, about $56 \% \mathrm{~K} 62 \mathrm{~T}$ variant of $T t \mathrm{HPr}$ was denatured, but about $19 \%$ for wild-type $T t \mathrm{HPr}$. These results indicated that the mutations led to less stable forms for these thermostable proteins, caused by the interruption of the salt-bridge in these proteins.

In conclusion, using high-quality atomic structures of homologous mesophilic and thermophilic HPrs, a detailed sequence and structural comparison was performed among five proteins. The salt-bridge was observed to play an important role in protein thermostability. Added mutations that interrupted the salt-bridges and subsequent thermo-stable measurements provided firm evidence that the salt-bridge was important in the thermostability. However, the details about other factors such as electrostatic interactions, self-buried core, and the influence of the value of ASA(R), still need further study. Most of time, thermostable proteins appear to rely on combinations of multiple stabilizing effects that manifest themselves in alteration of different habitat temperature.

We are grateful to Drs. Qi JianXun and Shi Yi for data collection and discussion. We thank Dr. Christopher Vavricka for his comments on the manuscript. This work was supported by the National High Technology Research and Development Program of China (Grant No. 2006BAD06A04) from Ministry of Science and Technology of China. George F. Gao is a leading principal investigator of the Innovative Research Group of the National Natural Science Foundation of China (Grant No. 80121003).

1 Xue Y, Xu Y, Liu Y, et al. Thermoanaerobacter tengcongensis sp. nov., a novel anaerobic, saccharolytic, thermophilic bacterium isolated from a hot spring in Tengcong, China. Int J Syst Evol Microbiol, 2001, 51: 1335-1341

2 Bao Q, Tian Y, Li W, et al. A complete sequence of the T. tengcongensis genome. Genome Res, 2002, 12: 689-700

3 Szilagyi A, Zavodszky P. Structural differences between mesophilic, moderately thermophilic and extremely thermophilic protein subunits: results of a comprehensive survey. Structure (London, England: 1993), 2000, 8: 493-504

4 Knapp S, de Vos WM, Rice D, et al. Crystal structure of glutamate dehydrogenase from the hyperthermophilic eubacterium Thermotoga maritima at 3.0 ̊̊ resolution. J Mol Biol, 1997, 267: 916-932

5 Karshikoff A, Ladenstein R. Ion pairs and the thermotolerance of proteins from hyperthermophiles: a "traffic rule" for hot roads. Trends Biochem Sci, 2001, 26: 550-556

6 Russell R J, Ferguson J M, Hough D W, et al. The crystal structure of citrate synthase from the hyperthermophilic archaeon Pyrococcus furiosus at $1.9 \AA$ resolution. Biochemistry, 1997, 36: 9983-9994

7 Sterner R, Liebl W. Thermophilic adaptation of proteins. Crit Rev Biochem Mol Biol, 2001, 36: 39-106

8 Vogt G, Woell S, Argos P. Protein thermal stability, hydrogen bonds, and ion pairs. J Mol Biol, 1997, 269: 631-643

9 Suvd D, Fujimoto Z, Takase K, et al. Crystal structure of Bacillus stearothermophilus alpha-amylase: possible factors determining the thermostability. J Biochem (Tokyo), 2001, 129: 461-468

10 Eijsink V G, Vriend G, Van der Zee J R, et al. Increasing the thermostability of the neutral proteinase of Bacillus stearothermophilus by improvement of internal hydrogen-bonding. Biochem J, 1992, 285: $625-628$ 
11 Tanner J J, Hecht R M, Krause K L. Determinants of enzyme thermostability observed in the molecular structure of Thermus aquaticus D-glyceraldehyde-3-phosphate dehydrogenase at 25 angstroms resolution. Biochemistry, 1996, 35: 2597-2609

12 Matsui I, Harata K. Implication for buried polar contacts and ion pairs in hyperthermostable enzymes. FEBS J, 2007, 274: 4012-4022

13 Bae E, Phillips G N Jr. Structures and analysis of highly homologous psychrophilic, mesophilic, and thermophilic adenylate kinases. J Biol Chem, 2004, 279: 28202-28208

14 Del Vecchio P, Elias M, Merone L, et al. Structural determinants of the high thermal stability of SsoPox from the hyperthermophilic archaeon Sulfolobus solfataricus. Extremophiles, 2009, 13: 461-470

15 Zhou X X, Wang Y B, Pan Y J, et al. Differences in amino acids composition and coupling patterns between mesophilic and thermophilic proteins. Amino Acids, 2008, 34: 25-33

16 Postma P W, Keizer H G, Koolwijk P. Transport of trehalose in Salmonella typhimurium. J Bacteriol, 1986, 168: 1107-1111

17 Cornilescu G, Lee B R, Cornilescu C C, et al. Solution structure of the phosphoryl transfer complex between the cytoplasmic A domain of the mannitol transporter IIMannitol and HPr of the Escherichia coli phosphotransferase system. J Biol Chem, 2002, 277: 4228942298

18 Weng Q P, Elder J, Jacobson G R. Site-specific mutagenesis of residues in the Escherichia coli mannitol permease that have been suggested to be important for its phosphorylation and chemoreception functions. J Biol Chem, 1992, 267: 19529-19535

19 Postma P W, Lengeler J W, Jacobson G R. Phosphoenolpyruvate: carbohydrate phosphotransferase systems of bacteria. Microbiol Rev, 1993, 57: 543-594

20 Azuaga A I, Neira J L, van Nuland N A. HPr as a model protein in structure, interaction, folding and stability studies. Protein Pept Lett, 2005, 12: 123-137

21 Jia Z, Quail J W, Waygood E B, et al. The 2.0-Å resolution structure of Escherichia coli histidine-containing phosphocarrier protein HPr. A redetermination. J Biol Chem, 1993, 268: 22490-22501

22 Herzberg O, Reddy P, Sutrina S, et al. Structure of the histidine-containing phosphocarrier protein HPr from Bacillus subtilis at 2.0-Å resolution. Proc Natl Acad Sci USA, 1992, 89: 2499-2503

23 Jia Z, Vandonselaar M, Hengstenberg W, et al. The $1.6 \AA$ structure of histidine-containing phosphotransfer protein HPr from Streptococcus faecalis. J Mol Biol, 1994, 236: 1341-1355

24 Pieper U, Kapadia G, Zhu P P, et al. Structural evidence for the evolutionary divergence of Mycoplasma from gram-positive bacteriathe histidine-containing phosphocarrier protein. Structure, 1995, 3: 781-790

25 Wittekind M, Rajagopal P, Branchini B R, et al. Solution structure of the phosphocarrier protein HPr from Bacillus subtilis by two-dimen- sional NMR spectroscopy. Protein Sci, 1992, 1: 1363-1376

26 Klevit R E, Waygood E B. Two-dimensional 1H NMR studies of histidine-containing protein from Escherichia coli. 3. Secondary and tertiary structure as determined by NMR. Biochemistry, 1986, 25: 7774-7781

27 Yin J, Liu Y, Li J, et al. Expression, crystallization and characterization of a novel 6-phospho- $\beta$-glucosidase from Thermoanaerobacter tengcongensis MB4. Chin J Biochem Mol Biol, 2008, 24: 916-924

28 Otwinowski Z, Minor W, Carter C W, et al. Processing of X-ray diffraction data collected in oscillation mode. Methods Enzymol, 1997, 276: $307-326$

29 Vagin A, Teplyakov A. MOLREP: an automated program for molecular replacement. J Appl Crystallogr, 1997, 30: 1022-1025

30 Murshudov G N, Vagin A A, Dodson E J. Refinement of macromolecular structures by the maximum-likelihood method. Acta Cryst D, 1997, 53: 240-255

31 Emsley P, Cowtan K. Coot: model-building tools for molecular graphics. Acta Cryst D, 2004, 60: 2126-2132

32 Adams P D, Grosse-Kunstleve R W, Hung L W, et al. PHENIX: building new software for automated crystallographic structure determination. Acta Crystallogr D Biol Crystallogr, 2002, 58: 19481954

33 Laskowski R A, MacArthur M W, Moss D S, et al. PROCHECK: a program to check the stereochemical quality of protein structures. J Appl Crystallogr, 1993, 26: 283-291

34 Barlow D, Thornton J. Ion-pairs in proteins. J Mol Biol, 1983, 168: $867-885$

35 Collaborative Computational Project, Number 4. The CCP4 suite: programs for protein crystallography. Acta Cryst D, 1994, 50: 760-763

36 Vriend G. WHAT IF: a molecular modeling and drug design program. J Mol Graph, 1990, 8: 52-56

37 DeLano W L. The PyMOL molecular graphics system. Palo Alto: DeLano Scientific. 2002

38 Razvi A, Scholtz J M. A thermodynamic comparison of HPr proteins from extremophilic organisms. Biochemistry, 2006, 45: 4084-4092

39 Ramachandran G N, Sasisekharan V. Conformation of polypeptides and proteins. Adv Protein Chem, 1968, 23: 283-438

40 Homeyer N, Essigke T, Ullmann G M, et al. Effects of histidine protonation and phosphorylation on histidine-containing phosphocarrier protein structure, dynamics, and physicochemical properties. Biochemistry, 2007, 46: 12314-12326

41 Li W F, Zhou X X, Lu P. Structural features of thermozymes. Biotechnol Adv, 2005, 23: 271-281

42 Ben M S, Aghajari N, Ben A M, et al. Enhancement of the thermostability of the maltogenic amylase MAUS149 by Gly312Ala and Lys436Arg substitutions. Bioresour Technol, 2011, 102: 1740-1746

Open Access This article is distributed under the terms of the Creative Commons Attribution License which permits any use, distribution, and reproduction in any medium, provided the original author(s) and source are credited. 